\title{
Die lange Dauer der Flucht - \\ Das Ankommen erleichtern durCh die Begleitung des Arbeitsmarktservice bis hin ZUR Arbeitsmarktintegration ${ }^{1}$
}

Marlies GatterbaUER, Arbeitsmarktservice (AMS), Wien

\section{INHALT}

1 Empirische Daten zur Arbeitsmarktsituation geflüchteter Menschen in Österreich

2 Das Ankommen durch die Unterstützung des AMS erleichtern......

2.1 Ausgewählte Betreuungs- und Vermittlungsangebote des AMS

3 Der Einsatz lohnt sich: Erfolge in der Integration von geflüchteten Menschen in den Arbeitsmarkt in Österreich - ein weiterer Schritt in Richtung „Ankommen“.

\section{Empirische Daten zur Arbeitsmarktsituation geflüchteter Menschen in Österreich}

„Flucht wird unter anderem als eine Reaktion auf Gefahren, (existentielle) Bedrohungen oder als unzumutbar empfundene Situationen beschrieben. Meist ist die Flucht ein plötzliches und eiliges, manchmal auch heimliches Verlassen eines Aufenthaltsorts oder Landes. Die eilige Bewegung weg von der Bedrohung ist oft ziellos und ungeordnet. “2

„Die lange Dauer der Flucht“ - dieser Titel wirft viele Fragen auf. Wann ist die Flucht vorüber? Ist sie es bei der Ankunft im Aufnahmeland? Nach der Zuerkennung eines Aufenthaltstitels? Bei der Aufnahme eines Jobs? Kann die Flucht jemals 1 Dieser Beitrag spiegelt die Datensituation zum Zeitpunkt seiner Erstellung Ende Oktober 2018 wider.

$2<$ https://de.wikipedia.org/wiki/Flucht> 
vorbei sein oder begleitet sie die geflüchteten Menschen ihr Leben lang im Kopf aufgrund von Erinnerungen an das Erlebte, aufgrund von Vorurteilen vonseiten der Aufnahmegesellschaft, mit denen sie oft konfrontiert werden, auch wenn sie bereits einen Aufenthaltstitel besitzen? Niemand kann ihnen die Erfahrungen, Erinnerungen und Schicksalsschläge, die sie auf ihrem langen Weg in ein sicheres Leben erfahren haben, abnehmen, aber ich möchte im Rahmen meiner Ausführungen einen Einblick in die Begleitung und Betreuung von geflüchteten Personen durch das AMS gewähren - eine Reise durch die Beratungs- und Betreuungswelt bis hin zur gelungenen Arbeitsmarktintegration. Dies ist der Beitrag, den das AMS als Organisation im Rahmen seiner Zuständigkeit leistet, um diesen Menschen eine Perspektive in ihrer neuen Heimat zu bieten und damit die Flucht vielleicht insofern ein Ende nehmen kann, indem diese Menschen beruflich ankommen können. Um sich ein Bild von der Arbeitsmarktsituation der geflüchteten Menschen in Österreich machen zu können, möchte ich mit einem kurzen Überblick über Zahlen, Daten und Fakten beginnen.

Das Arbeitsmarktservice (AMS) betreut Asylberechtigte und subsidiär Schutzberechtigte. Diese sind Österreicher/innen am Arbeitsmarkt gleichgestellt und benötigen keine Beschäftigungsbewilligung. Asylwerber/innen können sich grundsätzlich nicht beim AMS arbeitslos vormerken lassen oder vermittelt werden - ausgenommen Asylwerber/innen aus Syrien mit hoher Bleibewahrscheinlichkeit, die ab 1. Jänner 2018 am Integrationsjahr teilnehmen (AMS Produktblatt „Das Integrationsjahr“, Mai 2018). Für die Aufnahme einer Beschäftigung ist grundsätzlich eine Beschäftigungsbewilligung erforderlich. Sie wird Asylwerber/innen in aller Regel nur für Saisonarbeit im Gastgewerbe und in der Landwirtschaft für die Dauer von sechs Monaten erteilt (AMS Produktblatt „Beschäftigung von Asylwerber/innen“, September 2018).

Im Jahresdurchschnitt 2017 waren 29.182 Asylberechtigte und Personen mit subsidiärem Schutz ( +4.156 bzw. $+16,6 \%)$ beim AMS arbeitslos vorgemerkt oder haben eine Schulung des AMS besucht. Davon waren 61,7\% beim AMS Wien vorgemerkt. Der Anteil an allen beim AMS arbeitslos vorgemerkten Personen und Schulungsteilnehmer/ innen betrug 2017 7,1 \% (2016: 5,9\% und 2015: 4,1\%).

Mit September 2018 waren $31.218(+1.839$ bzw. $+6,3 \%)$ anerkannte Geflüchtete und subsidiär Schutzberechtigte beim AMS arbeitslos vorgemerkt oder haben eine Schulung des AMS besucht. Das sind 9,1\% aller arbeitslos vorgemerkten Personen und Schulungsteilnehmer/innen. 60,8\% von den 31.218 vorgemerkten Geflüchteten sind beim AMS Wien gemeldet. Die nachfolgende Grafik zeigt die Entwicklung des Bestandes anerkannter Geflüchteter und subsidiär Schutzberechtigter, die arbeitslos vorgemerkt oder in Schulung befindlich waren, seit Jänner 2014 für Österreich insgesamt:

Von den 31.218 geflüchteten Menschen, die beim AMS betreut werden, kommen 42,5\% aus Syrien, 21,0\% aus Afghanistan, 10,4\% aus Russland, 6,6\% aus dem Irak, 6,2\% aus dem Iran und 4,5\% aus Somalia. Der Großteil dieser Menschen, nämlich 54\%, befindet sich im Haupterwerbsalter (25 bis 44 Jahre), 28\% sind junge Menschen unter 25 Jahren und 18\% sind Personen ab 45 Jahren. 
Abb. 1: Entwicklung des Bestandes anerkannter Geflüchteter und subsidiär Schutzberechtigter, arbeitslos vorgemerkt oder in Schulung

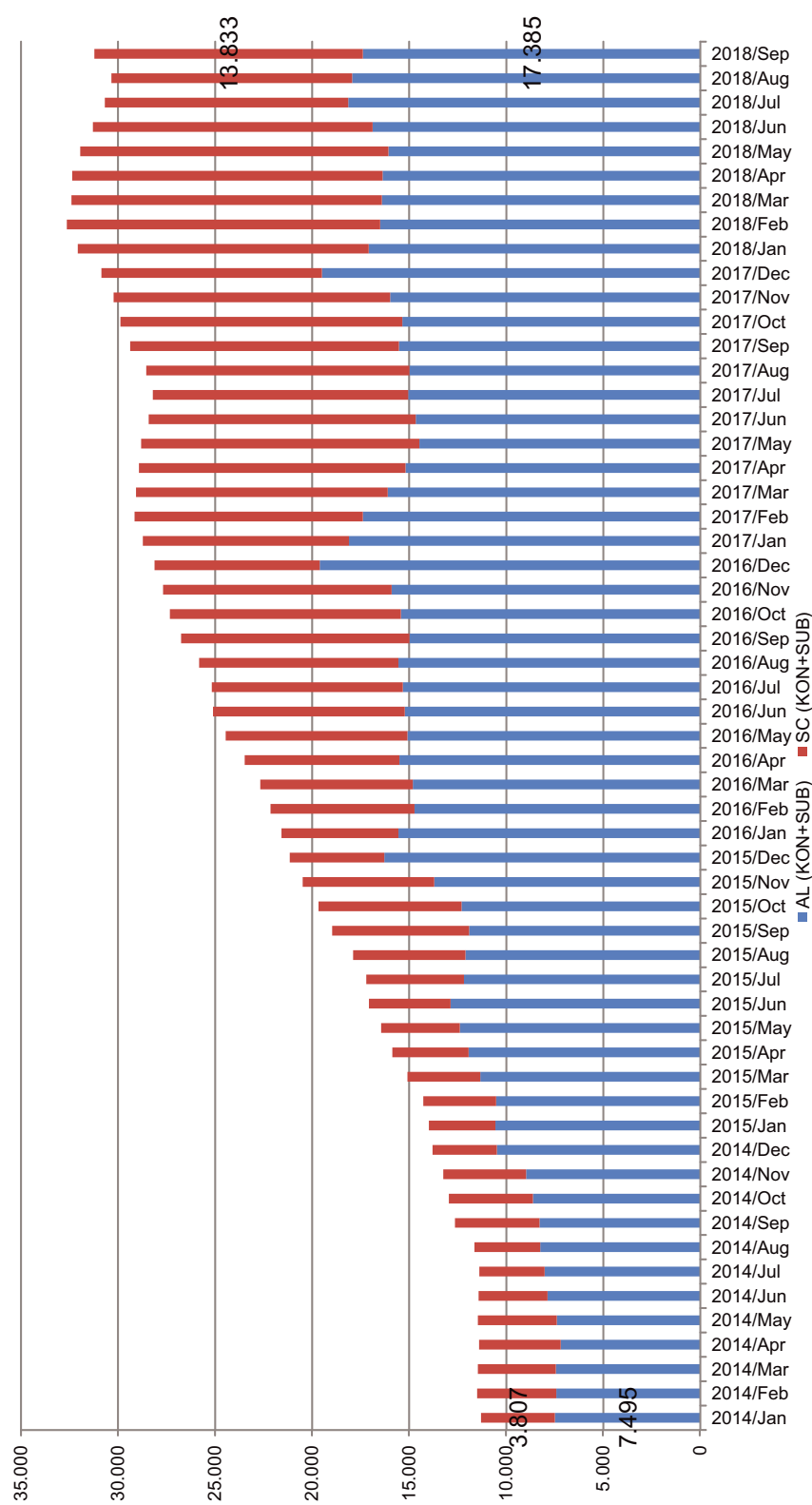

Quelle: AMS. 


\section{Das Ankommen durch die Unterstützung des AMS erleichtern}

Die Betreuung von geflüchteten Menschen ist mit einer ganzen Reihe von Herausforderungen verbunden. Dazu gehören noch fehlende Sprachkenntnisse, Qualifikationen, die noch aufgebaut werden bzw. an die Erfordernisse des österreichischen Arbeitsmarktes angepasst werden müssen. Im Fall, dass ein Anerkennungsverfahren von aus dem Herkunftsland mitgebrachten Qualifikationen notwendig ist, braucht es Zeit für das Verfahren, hinzu treten fehlende Netzwerke, Traumatisierungen sowie kulturelle Unterschiede, die ein Ankommen im Aufnahmeland erschweren. Außerdem gibt es nicht „,den/die Geflüchtete/n“, sondern jede geflüchtete Person bringt ihre eigene Geschichte, ihre eigenen Erfahrungen und Erlebnisse mit - all das gilt es im Umgang, in der Betreuung und in der Zusammenarbeit mit diesen Menschen zu berücksichtigen. Das Arbeitsmarktservice unterstützt hier durch seine Beratungs- und Vermittlungstätigkeiten, um diese Menschen sobald wie möglich in den Arbeitsmarkt in Österreich zu integrieren. Da fast $61 \%$ der beim AMS vorgemerkten geflüchteten Menschen im AMS Wien betreut werden, wird nachstehend ein Überblick insbesondere über dessen Angebote gegeben.

Das AMS Wien erweiterte 2017 das Angebot für Arbeitsuchende und eröffnete eine zentrale AMS-Erstberatungsstelle für Asyl- und subsidiär Schutzberechtigte, wel che am Standort des Österreichischen Integrationsfonds in Wien-Landstraße seit Mai 2017 betreut werden. Die Beratungsstelle bietet ihre Information und Beratung bei Bedarf in Arabisch, Farsi und Russisch an. Es profitieren dabei sowohl die Kund/inn/en als auch die Regionalen Geschäftsstellen des AMS Wien, da hier aufgrund der Sprachbarriere zeitaufwändige Beratung reduziert werden kann. Voraussetzungen für die Betreuung in der zentralen Erstberatungsstelle sind, dass die/der Kund/e/in asyl- oder subsidiär schutzberechtigt ist, sich erstmalig beim AMS meldet und über Deutschkenntnisse vom Niveau A1 verfügt. Personen, die noch nicht das Deutschniveau A1 erreicht haben, müssen einen Deutschkurs des Österreichischen Integrationsfonds (ÖIF) besuchen. Neben Deutschkursen werden Kompetenzchecks, Basisbildung, die Unterstützung bei der Anerkennung von mitgebrachten Qualifikationen, Berufsinformation und -orientierung, berufliche Aus- und Weiterbildung, Arbeitstrainings sowie Unterstützung bei der Jobsuche angeboten. Die Betreuung in der Erstberatungsstelle erfolgt für ein Jahr. Danach werden die Kund/inn/en von der regionalen Geschäftsstelle des Arbeitsmarktservice Wien betreut, die für ihren Wohnbezirk zuständig ist. Die Beratungsstelle ist für die Umsetzung des „Integrationsjahrgesetzes“ zuständig (AMS Produktblatt „Beratung für Asylberechtigte und subsidiär Schutzberechtigte“, September 2017; AMS Geschäftsbericht, 2017, S. 27). Mit dem Integrationsjahrgesetz (IJG), gültig ab 1.9.2017, wurde dem AMS die Abwicklung des Integrationsjahres übertragen. Asylberechtigte oder subsidiär Schutzberechtigte, die ihren Status nach dem 31.12.2014 zuerkannt bekommen haben und noch keinen Arbeitsplatz finden konnten, sind verpflichtet am Integrationsjahr teilzunehmen. Asylwerber/innen aus Syrien mit hoher 
Bleibewahrscheinlichkeit können unter bestimmten Voraussetzungen seit dem 1.1.2018 am Integrationsjahr teilnehmen. Weitere Voraussetzungen sind Deutschkenntnisse auf dem Sprachniveau A1, die erfüllte Schulpflicht und Arbeitsfähigkeit. Das Integrations jahr soll die Arbeitsmarktchancen durch die Erweiterung und den Erwerb von Kenntnissen in verschiedenen Berufen verbessern und unterstützt außerdem im Erlernen der deutschen Sprache. Das Integrationsjahr endet spätestens nach einem Jahr. Maßnahmen, die vor Ablauf des Jahres angefangen wurden, können noch abgeschlossen werden (AMS Produktblatt „Das Integrationsjahr"6).

\subsection{Ausgewählte Betreuungs- und Vermittlungsangebote des AMS}

In der Betreuung von Asylberechtigten und subsidiär Schutzberechtigten zielt das AMS auf eine nachhaltige Integration der Geflüchteten auf dem Arbeitsmarkt ab. Nachhaltige Integration bedeutet, den Geflüchteten mittelfristigeine stabile Beschäftigung auf dem österreichischen Arbeitsmarkt zu ermöglichen. Dafür ist es notwendig, ihnen eine entsprechend gute Qualifikation zu gewähren. Vorhandene Qualifikationen werden genützt und neben dem Spracherwerb entsprechend an die Erfordernisse des österreichischen Arbeitsmarktes angepasst. Entlang dieser strategischen Ausrichtung gestaltet sich das Maßnahmenangebot des AMS für Kund/inn/en mit Fluchthintergrund. Das Förderinstrumentarium des AMS ist breit gefächert und steht den anerkannten geflüchteten Menschen und subsidiär Schutzberechtigten zur Gänze zur Verfügung. Insbesondere werden dieser Zielgruppe folgende Maßnahmen angeboten:

- Kompetenzchecks,

- Deutschkurse,

- Unterstützungsleistungen von Betreuungs- und Beratungseinrichtungen insbesondere für die Anerkennung von Kompetenzen zwecks Erhöhung der Integrationsmöglichkeiten,

- Qualifizierungsangebote nach dem Kompetenzcheck etc.

Der Kompetenzcheck wurde im Zeitraum August bis Dezember 2015 durch das AMS Wien pilotiert und in der Folge umgesetzt. Im Laufe des Jahres 2016 wurde dieser nach und nach auf ganz Österreich ausgeweitet und wird seitdem von jeder Landesorganisation des AMS angeboten. Es gibt kein standardisiertes Format des Kompetenzchecks, sondern jede Landesorganisation führt diesen entsprechend den arbeitsmarktpolitischen Rahmenbedingungen des jeweiligen Bundeslandes durch. Am Kompetenzcheck des AMS Wien dürfen anerkannte geflüchtete Menschen und subsidiär Schutzberechtigte im Alter über 18 Jahren, mit einem Deutschniveau von mindestens A1 und mit Muttersprache Arabisch oder Farsi/Dari und einer Alphabetisierung in der lateinischen Sprache teilnehmen. Das AMS Wien bietet einen Kompetenzcheck für junge Männer im Alter zwischen 18 und 25 Jahren, einen für Männer über 25 Jahre und einen für Frauen über 18 Jahre an. Während die männlichen Teilnehmer/innen für die 
Dauer von fünf Wochen betreut werden, dauert der Kompetenzcheck für Frauen sieben Wochen. Die Teilnehmer/innen absolvieren wöchentlich zehn Stunden Workshops und eine Stunde Einzelcoaching. Im Rahmen der Workshops werden den Teilnehmer/inne/n österreichspezifische Kenntnisse, wie zum Beispiel über das Leben und Arbeiten in Österreich, das österreichische Bildungssystem, die österreichische Sozialversicherung, das Steuer- und Gesundheitssystem, das Alltagsleben, die Kultur etc., vermittelt. Das Einzelcoaching zielt insbesondere auf die Erhebung der im Ausland erworbenen formalen und beruflichen Kompetenzen sowie den Anerkennungsbedarf ab, um hier gezielt Unterstützung zu leisten.

Für Personen mit praktischer Berufserfahrung aus dem Herkunftsland aber ohne schriftlichen Nachweis darüber gibt es die Möglichkeit einer praktischen Erprobung ihrer theoretischen und praktischen Kompetenzen, welche entweder durch private Unternehmen oder Trainingsinstitute durchgeführt wird. Ist zum Beispiel eine Person als Tischler im Herkunftsland beschäftigt gewesen, dann wird bei der praktischen Erprobung überprüft, ob bzw. welche in Österreich verwendeten Werkzeuge oder Techniken bekannt sind oder nicht. Die durch den Kompetenzcheck gewonnenen Ergebnisse fließen in einen Ergebnisbericht ein, der einen unmittelbaren Berufswunsch, eine mittelbzw. langfristige Perspektivenplanung, eine detaillierte Dokumentation der erhobenen Kompetenzen sowie den Hinweis, ob eine praktische Erprobung erstellt wurde, beinhaltet. Die Ergebnisse der praktischen Erprobung werden in einem eigenen Ergebnisbericht festgehalten. Die Berichterstattung erfolgt an die/den zuständigen Berater/in und dient als Grundlage für die weiteren Schritte im Beratungsprozess.

Auf Basis der bis zum Ende des Jahres 2017 zur Verfügung stehenden Daten haben 14.050 Personen, davon 10.808 Männer (77\%) und 3.242 Frauen (23\%), am Kompetenzcheck teilgenommen. Davon stammten 20\% aus Afghanistan, 52\% aus Syrien, $6 \%$ aus dem Iran, $7 \%$ aus dem Irak und $15 \%$ aus anderen Herkunftsländern. 58\% der Teilnehmer/innen am Kompetenzcheck aus Syrien, 85\% jener aus dem Iran und 58\% jener aus dem Irak haben Matura oder ein Studium. Jedoch weisen nur 20\% der Teilnehmer/innen aus Afghanistan eine über die Pflichtschule hinausgehende abgeschlossene Ausbildung auf. Eine weitere Beobachtung unter den Teilnehmer/inne/n des Kompetenzchecks war, dass ein hoher Anteil der Frauen über ein Studium verfügt (27\%) (bei den Männern: 18\%).

Die ersten Ergebnisse über die Teilnehmer/innen des Kompetenzchecks wurden nach dem Pilotprojekt zu Beginn des Jahres 2016 veröffentlicht. Die darauf aufbauenden Erkenntnisse wurden durch die Studie „Displaced Persons in Austria Survey (DiPAS): Human Capital, Values, and Attitudes of Persons Seeking Refuge in Austria in 2015“, eine Studie des Wittgenstein Centre for Demography and Global Human Capital sowie eines Zusammenschlusses von Forschungseinrichtungen in und um Wien bestätigt. Bei DIPAS handelt es sich um eine unabhängige und freifinanzierte Forschung. ${ }^{3} \mathrm{Da}$ der Kompetenzcheck auch für andere europäische Arbeitsverwaltungen interessant

\footnotetext{
Vgl. <https://www.oeaw.ac.at/fileadmin/subsites/Institute/VID/PDF/Projects/DiPAS/wic dipas_2016_hintergrund_informationen.pdf $>$
} 
ist - dies spiegelt sich in dem Faktum wider, dass dieser bereits auf unterschiedlichen internationalen Konferenzen präsentiert werden durfte bzw. von interessierten europäischen Arbeitsverwaltungen besucht wurde -, scheint die Maßnahme auf der Website der Europäischen Kommission unter den PES Practices ${ }^{4}$ auf.

Das Jugendcollege ist ein gemeinsames Projekt des AMS Wien, des Fonds Soziales Wien und der Magistratsabteilung 17. Kofinanziert wird diese Initiative durch den Europäischen Sozialfonds (ESF). Das Projekt startete im August 2016 und ist auf 750 Teilnehmer/innen ausgerichtet. Das Jugendcollege ist für Asylwerber/innen und Asylberechtigte zwischen 15 und 21 Jahren gedacht, die nicht mehr schulpflichtig sind und deren Basiskenntnisse oder Fähigkeiten noch nicht ausreichen, um einen Arbeitsplatz zu finden oder im regulären Ausbildungssystem Fuß zu fassen. Im Rahmen der Betreuung im Jugendcollege erhalten die jungen Menschen einen individuellen Bildungsplan, Basisbildung (Deutsch, Mathematik, Englisch etc.), aber auch fachspezifische Module wie etwa IKT oder Werkstatt-Arbeit werden angeboten. Das Ziel des Projekts besteht darin, die Jugendlichen und jungen Erwachsenen so rasch wie möglich fit für ein eigenständiges Leben zu machen.

Im Projekt „b.mobile - Fachkräftepotenzial nutzen. Überregionale Lehrstellenvermittlung von jungen Erwachsenen mit Unterstützungsbedarf, insbesondere Asylberechtigten und subsidiär Schutzberechtigten“, das vom AMS Wien und der Wirtschaftskammer Österreich in Kooperation mit dem Wirtschafts- und Sozialministerium durchgeführt wird, werden junge Erwachsene, insbesondere Asylberechtigte und subsidiär Schutzberechtigte, bei der Aufnahme eines Lehrverhältnisses, vorrangig in den Bereichen Gastronomie, Handwerk und Technik, unterstützt. Zwischen Mai 2016 und März 2018 wurden im Rahmen dieses Projektes 340 Kursteilnehmer/innen verzeichnet, von denen 60 ein Lehrverhältnis und 30 ein Dienstverhältnis antraten. Gleichzeitig wurden rund 140 Praktika und 50 Schnuppertage akquiriert und absolviert (AMS Spezialthema, Juli 2018)

Die Lehre so wie auch die überbetriebliche Lehrausbildung werden als sehr wichtige Instrumente für die Integration von geflüchteten Menschen in den österreichischen Arbeitsmarkt gesehen. Für beim AMS vorgemerkte, angelernte oder ungelernte junge Erwachsene (ab dem 18. Lebensjahr) mit Pflichtschulabschluss wird die Facharbeiter/innen-Intensivausbildung (FIA) angeboten, welche diese auf eine Lehrabschlussprüfung vorbereitet. Diese Maßnahme dauert in der Regel zwischen 12 und 18 Monate und wird auch anerkannten Geflüchteten angeboten, für welche diese aufgrund von Deutsch- und Vorbereitungsmaßnahmen ein paar Monate länger dauert.

$4 \quad$ Vgl. $<$ http://ec.europa.eu/social/main.jsp?catld=1206\&langld=en $>$ 
3 Der Einsatz lohnt sich: Erfolge in der Integration von geflüchteten Menschen in den Arbeitsmarkt in Österreich - ein weiterer Schritt in Richtung „Ankommen“

Abb. 2: Entwicklung des Arbeitsmarkterfolgs zum jeweiligen Stichtag, Kontrollgruppe 1

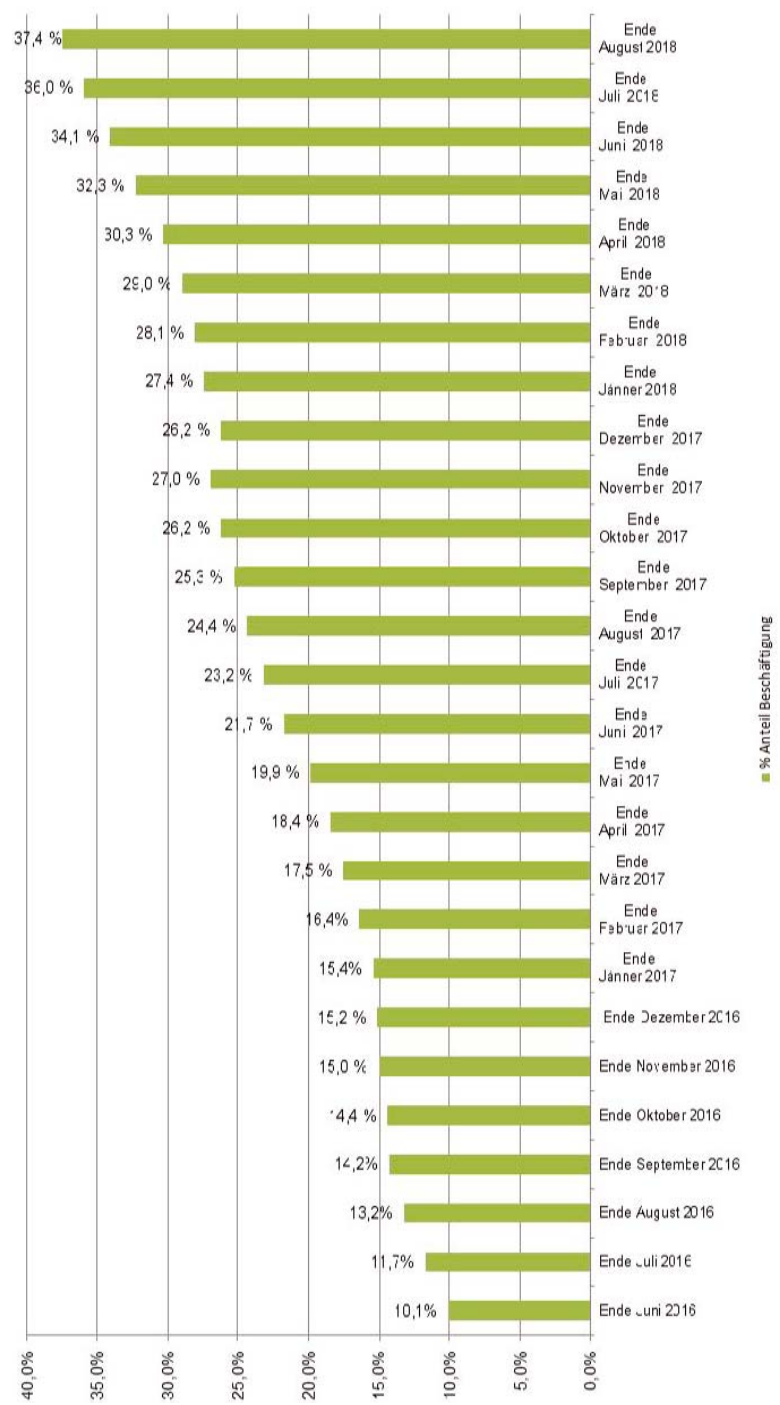

Quelle: AMS. 
Um Aussagen über den Erfolg in der Arbeitsmarktintegration treffen zu können, beobachtet das AMS seit Juni 2016 eine Gruppe von Personen, die im Jahr 2015 ihren Aufenthaltstitel erhielten und im Zeitraum Jänner 2015 bis Juni 2016 beim AMS arbeitslos vorgemerkt waren oder sich in Schulung befanden (ca. 9.500 Personen) (Kontrollgruppe 1). Von dieser Gruppe stand im Juni 2016 ein Anteil von 10,1\% in Beschäftigung, im August 2018 waren bereits 37,4\% beschäftigt (vgl. Abb. 2).

Bei der Kontrollgruppe 1 wird eine zusätzliche Kennzahl in Bezug auf die Nachhaltigkeit der Beschäftigungsaufnahmen ausgewertet. Von dieser Personengruppe konnten vom Jahresbeginn 2015 an 52,3\% ein unselbständiges oder selbständiges Beschäftigungsverhältnis aufnehmen, welches zumindest acht Tage gedauert hat und $45,5 \%$ konnten ein Beschäftigungsverhältnis aufnehmen, das zumindest 62 Tage gedauert hat. Ein Integrationserfolg ist auch aus dieser Kennzahl ableitbar.

Neben der Kontrollgruppe 1 wird eine zweite Kontrollgruppe (Kontrollgruppe 2; ca. 11.600 Personen) hinsichtlich des Arbeitsmarkterfolges monatlich beobachtet: Von jenen geflüchteten Menschen, die ihren Aufenthaltsstatus im Jahr 2016 erhalten haben und im Zeitraum Jänner 2016 bis Juni 2017 beim AMS arbeitslos vorgemerkt oder in Schulung waren, standen im Juni 2017 11,4\% in Beschäftigung, im August 2018 traf dies bereits auf 29,9\% zu (vgl. Abb. 3).

Abb. 3: Entwicklung des Arbeitsmarkterfolgs zum jeweiligen Stichtag, Kontrollgruppe 2

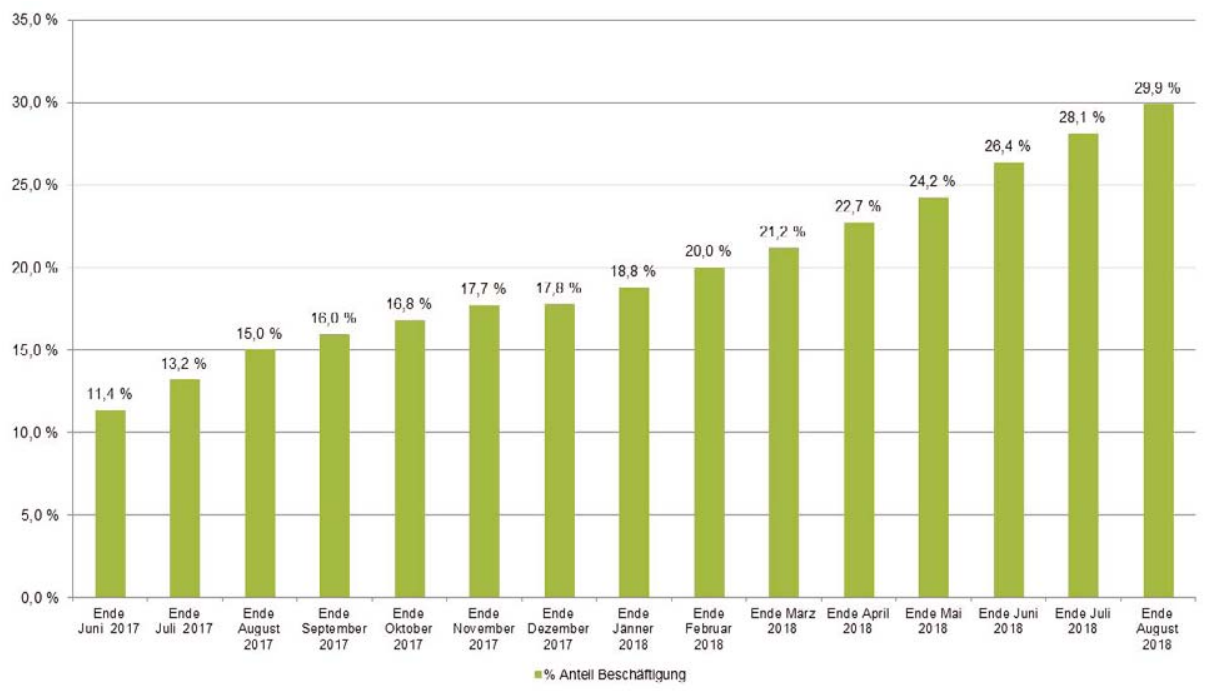

Quelle: AMS. 
Angelehnt an eine Prognose des deutschen Instituts IAB (Institut für Arbeitsmarktund Berufsforschung $)^{5}$, welches für Deutschland eine Prognose erstellte, dass innerhalb von 5 Jahren 50 Prozent der Asylberechtigten in den Arbeitsmarkt integriert werden könnten, kann das AMS mit den bisherigen Erfolgen in der Arbeitsmarktintegration von geflüchteten Menschen sehr zufrieden sein.

Das AMS kann nur die Arbeitsmarktintegration der beim AMS gemeldeten Kund/ inn/en beurteilen. Die Integration in den Arbeitsmarkt erfolgt aber auch außerhalb des AMS, insbesondere mit Hilfe der Communities oder vorhandener Netzwerke. Für die Beobachtung der Erfolge in der Arbeitsmarktintegration wird regelmäßig auch die Zahl der unselbständig Beschäftigten aus den wichtigsten Herkunftsländern wie Syrien und Afghanistan herangezogen. 42,6\% der insgesamt beim AMS im September 2018 als arbeitslos vorgemerkten oder in Schulung befindlichen anerkannten Geflüchteten und subsidiär Schutzberechtigten stammten aus Syrien und 21,0\% aus Afghanistan. Ende September 2018 standen laut dem Hauptverband der österreichischen Sozialversicherungsträger 7.619 Syrer/innen und 9.334 Afghan/inn/en in unselbständiger Beschäftigung. Das waren somit um $+70,6 \%$ (Syrer/innen) und $+38,3 \%$ (Afghan/inn/ en) mehr als noch im September 2017. Die Sozialversicherung speichert keine Daten in Bezug auf den Aufenthaltstitel, somit sind in diesen Zahlen auch Syrer/innen und Afghan/inn/en enthalten, die nicht als Geflüchtete nach Österreich gekommen sind. Ein Datenquercheck ergab jedoch, dass ein Großteil dieser beschäftigten Personen in der Grundversorgung war und somit Fluchthintergrund angenommen werden kann. Aus den Daten wird klar ersichtlich, dass eine beträchtliche Zahl von Menschen ohne Mitwirkung des AMS durch Eigeninitiative mit Hilfe ihres Netzwerkes und ihrer Community selbständig einen Job findet.

\section{Fazit}

Trotz der bestehenden Herausforderungen verläuft die Arbeitsmarktintegration von geflüchteten Personen aufgrund der derzeit guten konjunkturellen Lage, des umfassenden und strukturierten Vermittlungs- und Betreuungsangebotes des AMS und der Eigeninitiative dieser Menschen sehr erfolgreich und zufriedenstellend. Wie die Ergebnisse zeigen, liegen wesentliche Voraussetzungen, um die lange Dauer der Flucht etwas zu begrenzen, in einer raschen Arbeitsmarktintegration, einer sinnstiftenden Beschäftigung sowie einem entsprechenden Einkommen.

\footnotetext{
Vgl. $<$ http://www.iab.de/>
} 


\section{Bibliographie}

Bundesministerium für Arbeit, Soziales und Konsumentenschutz (BMASK) (ed.) (2009, 2010), Mitwirkung an den Broschüren „Arbeitsmarktpolitik im Jahr 2009“ und „Arbeitsmarktpolitik im Jahr 2010“.

$<$ https://broschuerenservice.sozialministerium.at/Home/Download?publicationId=33>

$<$ https://broschuerenservice.sozialministerium.at/Home/Download?publicationId=34>

Fischer M. \& Gatterbauer M.(2010), Gender Budgeting, ein Leitfaden zur Umsetzung der Haushaltsrechtsreform. Bundesministerium für Finanzen, Working Paper 2.

Rengs B., Buber-Ennser I., Kohlenberger J., Hoffmann R., Soder M., Gatterbauer M., Themel K. \& Kopf J. (2017), Labour market profile, previous employment and integration potential of refugees from Syria, Iraq and Afghanistan: An Austrian case study. VID Working Paper 13. 